\title{
Electroless Deposition of Cu-SWCNT Composites
}

\author{
Pavan M. V. Raja ${ }^{1}$, Gibran L. Esquenazi ${ }^{1}$, Daniel R. Jones ${ }^{2}$, Jianhua Li ${ }^{3}$, Bruce E. Brinson ${ }^{1}$, \\ Kourtney Wright ${ }^{1}$, Cathren E. Gowenlock ${ }^{2, *}$ and Andrew R. Barron ${ }^{1,2,4, * \mathbb{C}}$ \\ 1 Department of Chemistry, Rice University, Houston, TX 77005, USA; vr15@rice.edu (P.M.V.R.); \\ gibranesquenazi@gmail.com (G.L.E.); brinson@rice.edu (B.E.B.); kourtney.d.wright@lmco.com (K.W.) \\ 2 Energy Safety Research Institute, Swansea University, Bay Campus, Swansea SA1 8EN, UK; \\ d.r.jones@swansea.ac.uk \\ 3 Shared Equipment Authority, Rice University, Houston, TX 77005, USA; Jianhua.Li@rice.edu \\ 4 Department of Materials Science and Nanoengineering, Rice University, Houston, TX 77005, USA \\ * Correspondence: c.gowenlock@swansea.ac.uk (C.E.G.); a.r.barron@swansea.ac.uk (A.R.B.); \\ Tel.: +44-01792-606930 (A.R.B.)
}

Received: 29 August 2019; Accepted: 2 October 2019; Published: 7 October 2019

\begin{abstract}
In this work, as-received HiPCO single walled carbon nanotubes (SWCNTs) are incorporated in a controllable manner at various concentrations into Cu-SWCNT composites via electroless plating, by varying the related reaction times, with polyethylene glycol (PEG) used as a dispersing agent. The resultant samples were analyzed using scanning electron microscopy (SEM) for morphology assessment, energy dispersive X-ray analysis (EDX) and X-ray photoelectron spectroscopy (XPS) for elemental analysis, X-ray diffraction (XRD) for the assessment of crystal phase identification, and Raman spectroscopy for the confirmation of the presence of the incorporated SWCNTs. The Cu-SWCNT composites were found to contain carbon, catalytic iron (associated with the raw, as-received SWCNTs), oxygen, and copper; the latter was found to be inversely proportional to carbon and iron contents. The oxygen (associated with both the SWCNT defect sites and oxidized copper surfaces) remained more or less constant regardless of the proportion of SWCNTs in the composites. The Raman $\mathrm{I}_{\mathrm{G}}: \mathrm{I}_{\mathrm{D}}$ ratio remains within the experimental error constant, indicating that the electroless deposition does not have a deleterious effect on the SWCNTs. At short deposition times, SEM revealed a relatively dense structure comprising a distinctive fibrous morphology, suggestive of an underlying SWCNT substrate coated with copper; however, with increasing deposition, a more porous morphology is observed. The size of the granular particles increases up until $10 \mathrm{~min}$ of reaction, after which time it remains unchanged.
\end{abstract}

Keywords: single walled carbon nanotube; SWCNT; copper; electroless; coating

\section{Introduction}

There has been significant recent interest in the formulation of composites comprising carbon nanotubes (CNTs) imbedded in a copper matrix. The rationale for this composition is to combine the strength, low weight and conductivity of the CNTs with the conductivity and processability of copper, in particular in wires and cables [1-3]. While undoped CNT fibers show lower electrical conductivity than that of copper, individual CNTs have been reported to have higher ampacity [4,5]. In general, simultaneous high ampacity and conductivity in a single material is difficult to achieve; however, by incorporating $\mathrm{CNTs}$ into $\mathrm{Cu}$ wires, it may be possible to obtain a composite material with high conductivity as well as high ampacity $[1,6,7]$. The resulting composite is often termed "ultra-conductive copper". Such a composite material can potentially be used in electrical applications (e.g., wires and cables for the transmission of large amounts of electrical energy) [8]. 
The fabrication of $\mathrm{Cu}-\mathrm{CNT}$ composites has been achieved via a number of synthetic routes [9-11]; however, Subramaniam et al. reported the best results to date for a sample with a conductivity of $4.7 \times 10^{5} \mathrm{~S} / \mathrm{cm}$ and an ampacity of $630 \times 10^{6} \mathrm{~A} / \mathrm{cm}^{2}$, which is a 100-fold increase in the ampacity of the $\mathrm{Cu}-\mathrm{CNT}$ composite versus $\mathrm{Cu}[6,7]$, although the reported ampacity may be artificially high due to heat transfer from the short test structure $(50 \mu \mathrm{m})$ to the contacts and the substrate [4]. Nevertheless, they proposed that a controlled homogeneous seeding of $\mathrm{Cu}$ onto the CNT surfaces is key to their results [6]. As a consequence, our research group and others have investigated the seeding of $\mathrm{Cu}$ crystals on the surface of CNTs; in particular, the effect of surface functionality [12-14]. During our studies [12], we showed that electroless deposition results in the coating of individual and/or small bundles of single walled carbon nanotubes (SWCNTs) to create a homogeneous composite; however, the deposition is facilitated by the functionalization of the SWCNT surface. Hannula et al. have shown a similar dependence on functionalization for MWCNTs [15].

Copper-multiwalled carbon nanotube (MWCNT) nanocomposite powders have been reported [10,16-19]. In all cases, $\mathrm{Cu}$ is deposited on the surface of MWCNTs; however, with a sufficient MWCNT diameter, $\mathrm{Cu}$ is also deposited on the inside [17]. The homogeneity of the resulting composite was either promoted by physical milling post-deposition [18], acid treatment of the MWCNTs [17], the use of an anionic dispersant of sodium dodecyl sulfate (SDS) [20], and polymeric dispersants such as hydroxypropyl cellulose (HPC) [20]. Double-walled carbon nanotube (DWCNT) copper composite wires have been reported with only a $12 \%$ decrease in conductivity as compared to analogous copper wires, but with a $10 \%$ increase in tensile strength [21]. Despite these reports, there is a paucity of studies focused on Cu-SWCNT composite formation in electroless bath conditions.

We have recently reported the electromigration of raw (as synthesized) SWCNTs [22] and the formation of Cu-SWCNT composites by electrodeposition [23] in the absence of dispersing agents. During these studies we observed that dispersion in the aqueous bath was important for uniform $\mathrm{Cu}-\mathrm{SWCNT}$ distribution. In this regard, we report herein the electroless deposition of Cu-SWCNT composites using polyethylene glycol (PEG) as a dispersant. Our study can potentially guide further experimentation pertaining to the development and reliable scale-up of $\mathrm{Cu}$-SWCNT composites for electrical applications, and metal-nanomaterial composites in general, for additional uses. This work also further builds on an earlier copper-CNT study involving electroless deposition and serves to provide a comparison between the material characteristics of $\mathrm{Cu}$-SWCNT composites generated via electroless and electroplating methods, and the use-or absence of-dispersing agents for the SWCNTs.

\section{Materials and Methods}

Unpurified raw HiPCO (high pressure carbon monoxide process) SWCNTs (SWCNTs) were obtained from Tubes@Rice (Houston, TX) batch 09-HiPco-0093 (batch no. 188.4) and used without further purification. All SWCNT handling was carried out by previously published methods [24]. Polyethylene glycol (PEG, $M_{\mathrm{W}}=8000$ Daltons), disodium ethylenediamine tetraacetate $\left(\mathrm{Na}_{2} \mathrm{EDTA}\right)$, $\mathrm{CuSO}_{4} \cdot 5 \mathrm{H}_{2} \mathrm{O}$, formaldehyde, sodium sulfate, sodium hydroxide, and formic acid were obtained from Sigma Aldrich (St Louis, MO). Aqueous acetic acid ( $0.8 \mathrm{M})$, acetone and ethanol were obtained from Fisher Scientific (St. Louis, MO) and used as is. Polyvinylidene fluoride (PVDF) membranes were purchased from Millipore (Burlington, MA).

SWCNTs were plated with copper in an electroless bath for varying durations to determine the effect of reaction time on the ratio between the two materials. The bath composition was adapted from our previously used methodology $[12,25]$ and the formulation used by Caturla et al. in applying electroless nickel coatings to graphite [26]. The electroless reactions were conducted as follows: raw HiPCO SWCNT $(48 \mathrm{mg})$ were sonicated in DI water $(96 \mathrm{~mL})$ with polyethylene glycol $(6.6 \mathrm{~g})$ for $1 \mathrm{~h}$ to prepare a stock SWCNT dispersion for use in the electroless plating experiments. A sample of the stock dispersion (16 mL, equivalent to $8 \mathrm{mg}$ SWCNT per aliquot) was used for each reaction batch. Preparing the stock SWCNT dispersion in a PEG solution (with the PEG acting as a nonionic surfactant) eliminated the need to weigh out the hard-to-disperse SWCNTs and enabled the better control of 
nanotube dosage into the reaction mixture. Copper for electroless plating was sourced through copper sulfate salt, while disodium EDTA was used as the chelating agent. Polyethylene glycol was used as a stabilizer, formaldehyde was used as a reducing agent, and sodium sulfate was applied to enhance the copper plating rate. Sodium formate served as a buffer and also as a brightener [27]. Sodium hydroxide was added to ensure that the bath remained alkaline [28]. Copper sulfate pentahydrate $(0.5 \mathrm{~g}), \mathrm{Na}_{2}$ EDTA $(2.3 \mathrm{~g})$, sodium sulfate $(1 \mathrm{~g})$, sodium carbonate $(0.8 \mathrm{~g})$, and polyethylene glycol $(13.2 \mathrm{~g})$ were added along with DI water $(90 \mathrm{~mL})$ in a $200 \mathrm{~mL}$ beaker and heated to $\sim 90{ }^{\circ} \mathrm{C}$ for at least $20 \mathrm{~min}$ with stirring at $700 \mathrm{rpm}$ until the bath turned a dark blue. The dark blue coloration is likely due to the formation of an EDTA-copper complex [29]. Then, aqueous $\mathrm{NaOH}(35 \mathrm{~mL}, 1 \mathrm{M})$, formaldehyde $(24 \mathrm{~mL}$, $30 \%)$, formic acid $(0.6 \mathrm{~mL})$, and an aliquot of raw SWNTs dispersed in PEG (16 mL, see above) were added to the bath (equivalent of $8 \mathrm{mg}$ SWCNT) and stirred for the desired reaction time (see Table 1) with continued heating $\left(94^{\circ} \mathrm{C}\right)$, at $1200 \mathrm{rpm}$. After each reaction, the contents of the bath were divided into two aliquots and filtered onto two separate PVDF membranes (after taring the membranes to determine the actual mass of filtrate obtained on them). After weighing, the contents of both filter membranes were transferred to a tared sample vial and air dried for further characterization.

Table 1. Composition of electroless bath, reaction time, and yield of products. SWCNT: single walled carbon nanotube.

\begin{tabular}{ccccc}
\hline $\begin{array}{c}\text { Reaction Volume } \\
(\mathbf{m L})\end{array}$ & $\begin{array}{c}\text { SWCNT } \\
(\mathbf{m g})\end{array}$ & $\begin{array}{c}\text { Reaction Time } \\
(\mathbf{m i n})\end{array}$ & $\begin{array}{c}\text { Air-Dried Product } \\
(\mathbf{m g})\end{array}$ & SWCNT (wt\%) $\mathbf{1}^{\mathbf{2}}$ \\
\hline 140 & 8 & 5 & 75.2 & 10.6 \\
140 & 8 & 15 & 95.5 & 8.3 \\
140 & 8 & 30 & 111.0 & 7.2 \\
140 & 8 & 45 & 123.2 & 6.5 \\
\hline
\end{tabular}

\footnotetext{
$\overline{{ }^{1} \text { Note that the wt.\% values in this column are calculated ideal values based on the mass of SWCNT added to the }}$ electroless reaction mixtures, as a function of the final mass of the air-dried products from the reactions. These values are used subsequently for reference only and should not be confused with the actual wt.\% and at.\% data as determined via energy dispersive X-ray spectroscopy (EDX), X-ray photoelectron spectroscopy (XPS), and X-ray diffraction (XRD).
}

The electroless Cu-SWCNT composites were characterized using a variety of material characterization techniques. Scanning electron microscopy (SEM) was performed on a FEI Quanta 400 ESEM FEG. The instrument also included an energy dispersive X-ray spectroscopy (EDX) capability. Samples were affixed on carbon tape, onto aluminum SEM stubs, which were used as received. An operating voltage of $20 \mathrm{kV}$ was typically used, and the samples were imaged at a $10 \mathrm{~mm}$ working distance (with a spot size of $3 \mu \mathrm{m}$ ), under high vacuum conditions. Granular particle sizes were calculated by visually estimating the diameters of the particles on the SEM images using the scale bar and the length of a line drawn across randomly selected individual particles in a PowerPoint slide containing the SEM image. The evaluated numbers are purely for the purpose of identifying trends and are not absolute values considering the random nature of the electroless process and the heterogeneity of the SWCNT stating material. Cubic structures were again visually counted (no software was used) from SEM images. A few images (typically $\geq 3$ per sample type) were averaged in this manner to obtain the counts. EDS data was acquired using the SEM's EDS detector, and EDAX TEAM ${ }^{\mathrm{TM}}$ software was used to process the acquired data from three sample locations. X-ray photoelectron spectroscopy (XPS) data was acquired on a sample area measuring $700 \mu \mathrm{m} \times 300 \mu \mathrm{m}$ via a Kratos Axis Supra system with the following settings: Al- $\mathrm{K}_{\alpha} \mathrm{X}$-ray source $(1486.7 \mathrm{eV})$, a $15.00 \mathrm{~mA}$ emission current, and $20 \mathrm{eV}$ pass energy. Each sample was subjected to charge neutralization via a $1 \mathrm{~V}$ filament bias, 0.4 A filament current, and 3.3 V charge balance. A dwell time of $250 \mathrm{~ms}$ (single sweep) was used to record the data. CasaXPS software (Version 2.3.12.8) was used to analyze the recorded data. Spectrum energy calibration was conducted for the $\mathrm{C}$ 1s peak at binding energy of $284.8 \mathrm{eV}$ (as per the NIST XPS database). Multiplex spectra for various elemental energy levels (C 1s, O 1s, and Cu 2p) were acquired to yield insights on speciation and variation of composition in the samples. A Rigaku 
D/MAX 2100 diffractometer was used ( $\mathrm{Cu}-K_{\alpha}$ radiation), with a graphite-based monochromator to obtain X-ray diffraction (XRD) measurements. Single crystal silicon (511) wafer samples holders were employed in the case of all samples to ensure zero-background. Rigaku PDXL2 software was used to analyze the obtained data. The Reitveld refinement of obtained XRD data was used to quantify $\mathrm{Cu}$ (PDF 04-009-2090) and $\mathrm{Cu}_{2} \mathrm{O}$ (PDF 04-002-3214) in the samples. A Raman analysis of samples was conducted using a Renishaw inVia Raman Microscope (Wotton-under-Edge, UK) with the following settings: a $514.5 \mathrm{~nm}$ wavelength setting, 50× LWD magnification, in the range of $100 \mathrm{~cm}^{-1}$ to $3300 \mathrm{~cm}^{-1}$. The data were subjected to background subtraction and baselining, and $\mathrm{I}_{\mathrm{G}} / \mathrm{I}_{\mathrm{D}}$ ratios were calculated and averaged over three repeat trials.

\section{Results and Discussion}

Table 1 summarizes the reaction conditions used to obtain the various $\mathrm{Cu}-\mathrm{SWCNT}$ electroless samples. It should be noted that since raw (unpurified) SWCNTs were employed, the weight \% included the residual catalytic iron impurity (20-47 wt.\%) and minor amounts of amorphous carbon [30-32]. Hence, the actual proportion of pure SWCNTs was at least correspondingly lower after discounting the impurities. The choice of raw as opposed to purified SWCNTs was based upon recent results that showed the ultimate conductivity of a Cu-CNT composite is only slightly affected by the presence of residual catalyst [33]. It is also likely that the air-drying process caused the samples to retain small residual amounts of moisture, but the samples were subsequently stored in airtight containers prior to characterization.

Figure 1 shows the mass of content increases with time. The mass of copper deposited increases at an exponential rate. Electroless deposition is ordinarily an autocatalyzed process [34]; however, this would be expected to result in a constant reaction rate, until $\mathrm{Cu}^{2+}$ depletion in the bath occurs. Reactions of this type have the property that the rate equations are nonlinear; that is, the reaction is very slow in the beginning, but steadily increases as more product is formed. However, as is clear from Figure 1, this is not the case, since there is clearly no induction period. We note that the deposition does not follow either first- or second-order rate reaction kinetics. It has been observed that the electroless deposition of $\mathrm{Cu}$ onto CNTs is facilitated by the surface functionality of the CNTs $[12,15]$. This can be associated with the inherent oxygen species present during the synthesis and purification of the CNTs [12], or due to the attachment of specific functional groups that lower the reduction potential of $\mathrm{Cu}^{2+}$ upon coordination $[15,25]$. At long deposition times, stagnation in the mass of copper deposited could likely also be due to the depletion of copper ions from the plating bath.

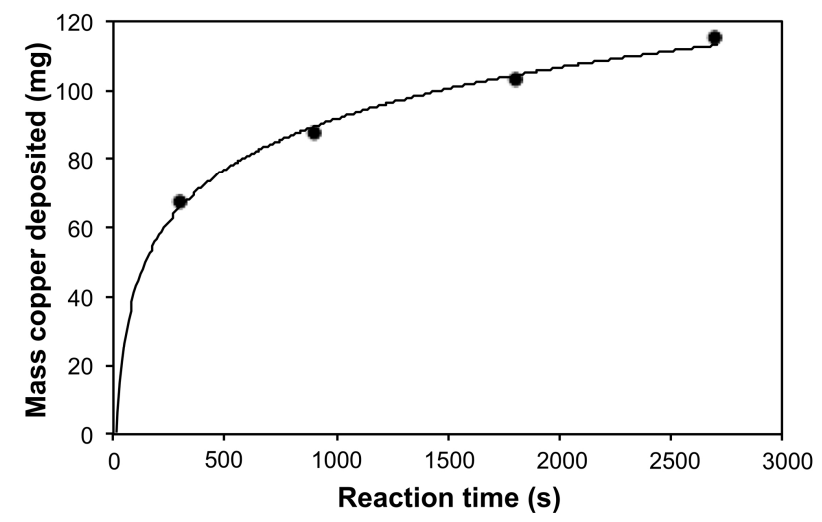

Figure 1. Plot of copper mass (mg) in the resulting Cu-SWCNT composites grown by electroless deposition as a function of reaction time $(\mathrm{s})\left(R^{2}=0.99\right)$.

\subsection{SEM Analysis}

SEM images of SWCNT-metal composites from selected electroless reaction conditions are shown in Figure 2. For the product formed after $5 \mathrm{~min}$ (10.6 wt.\% SWCNTs) SEM revealed a relatively dense 
structure (Figure 2a) comprising a distinctive fibrous morphology clearly seen at higher magnifications (Figure 2b,c) that is suggestive of an underlying SWCNT substrate coated with copper. A similar morphology is observed in the electroless coating of SWCNTs with CdS and CdSe [35]. In addition to granular particles, there are a few cubic crystals typical of $\mathrm{Cu}$ growth [12]. As the reaction time increases, a more porous morphology is observed (Figure $2 \mathrm{~d}, \mathrm{~g}, \mathrm{j}$ ). In addition, the prevalence of the cubic crystals increases dramatically, and the overall structure loses the underlying fibrous substructure (Figure 2f,i,l). Although the individual SWCNTs (or bundles thereof) are not observed after $10 \mathrm{~min}$ of deposition, their presence is confirmed by Raman spectroscopy (see Section 3.5).

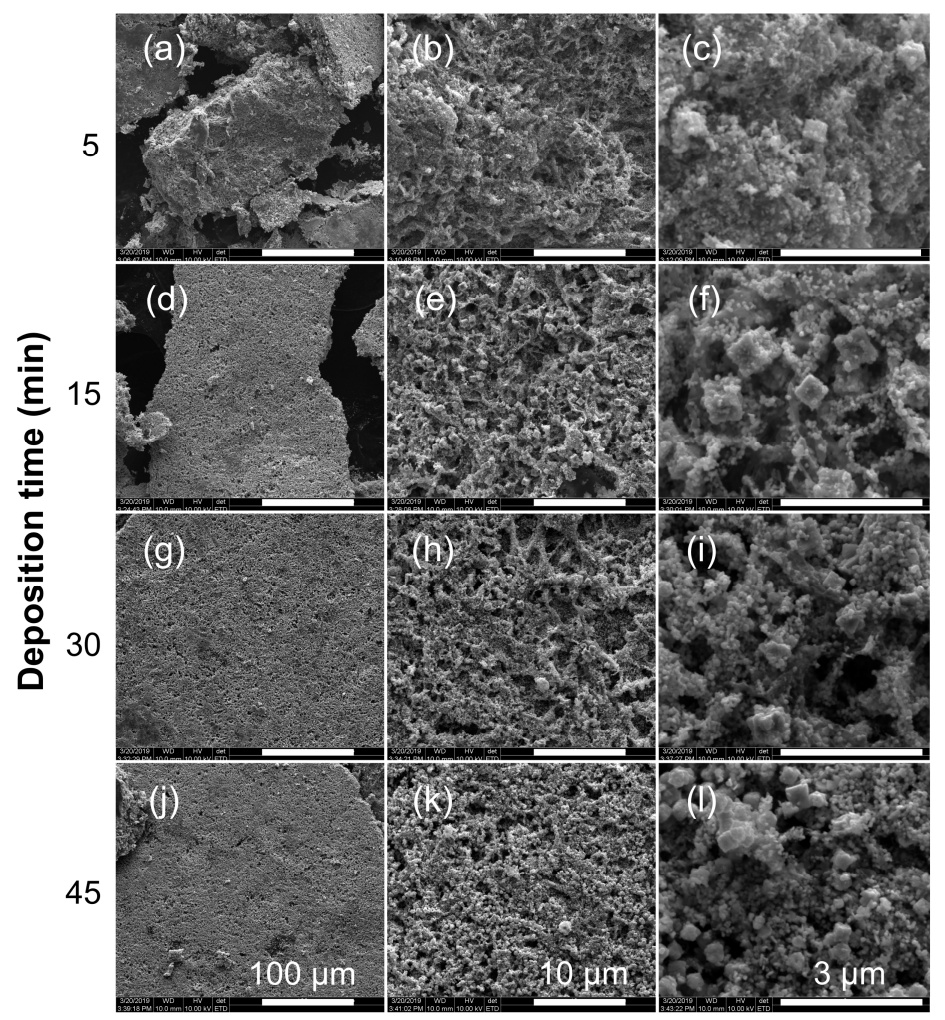

Figure 2. Representative SEM images of electroless Cu-SWCNT composites deposited (at a bath temperature of $\sim 90^{\circ} \mathrm{C}$ ) at different reaction times with resulting SWCNT composition: (a-c) $5 \mathrm{~min}$, 10.6 wt.\% SWCNTs; (d-f) 15 min, 8.33 wt.\% SWCNTs; (g-i) 30 min, 7.2 wt.\% SWCNTs; (j-1) 45 min, 6.5 wt. $\%$ SWCNTs. Scale bar $=100 \mu \mathrm{m}(\mathrm{a}, \mathrm{d}, \mathrm{g}, \mathrm{j}), 10 \mu \mathrm{m}(\mathrm{b}, \mathrm{e}, \mathrm{h}, \mathrm{k})$, and $3 \mu \mathrm{m}(\mathrm{c}, \mathrm{f}, \mathrm{i}, \mathrm{l})$.

Table 2 provides a comparison of the morphologies between the various samples analyzed from multiple SEM images. The size of the granular particles increases until $10 \mathrm{~min}$ of reaction, after which time it remains unchanged. This could have been due to the smoothening of particle surfaces (thereby decreasing the number of active sites or surface area available on each particle) with increasing deposition beyond $10 \mathrm{~min}$ that likely caused a self-limitation of granular particle size, coupled with the vigorous stirring of the reaction mixture, which did not allow further physical deposition or the mechanical bonding of material on each particle. Mechanical bonding, in conjunction with chemical deposition, has been postulated in prior research [36] to explain the electroless deposition of copper particles on ceramic surfaces. By comparison, the abundance of the cubic crystals increases exponentially (Figure 3), although the range of their sizes (as measured by one face of the cubic crystal by SEM) remains constant. Again, the size limitations of cubic crystals could have been due to a combination of the stirring conditions used with the reaction mixtures. The relative increase of the number of cubic particles suggests an initial deposition of $\mathrm{Cu}_{2} \mathrm{O}$ granular materials that later shifted toward cubic copper crystals, as later evidenced by XRD data. Thus, as copper deposition increases with time, the size of both the granular and cubic morphology remains consistent, but the 
relative amount of the different morphologies changes (Table 2). Considering the various reaction parameters involved (e.g., mixing speed, temperature, proportions of the various reactants, etc.), further research is needed before finalizing a viewpoint on the abundance and sizes of granular and cubic crystalline phases.

Table 2. Morphological composition of Cu-SWCNT composites prepared by electroless deposition as a function of reaction time.

\begin{tabular}{|c|c|c|c|c|}
\hline $\begin{array}{l}\text { Reaction Time } \\
\text { (min) }\end{array}$ & $\begin{array}{l}\text { SWCNT } \\
\left(w t^{\circ}\right)^{1}\end{array}$ & $\begin{array}{l}\text { Granular Particle } \\
\text { Size }(\mathrm{nm})^{2}\end{array}$ & $\begin{array}{c}\text { Abundance of } \\
\text { Cubic Structures }\end{array}$ & $\begin{array}{l}\text { Size of Cubic } \\
\text { Crystal }(\mathrm{nm})^{2}\end{array}$ \\
\hline 5 & 10.6 & $\sim 70$ & 8 & $150-600$ \\
\hline 15 & 8.3 & 70-130 & 19 & $290-550$ \\
\hline 30 & 7.2 & 70-130 & $43-52$ & $350-570$ \\
\hline 45 & 6.5 & 70-130 & $>200$ & $160-490$ \\
\hline
\end{tabular}

1 The wt.\% values in this column are calculated ideal values based on the mass of SWCNT added to the electroless reaction mixtures, as a function of the final mass of the air-dried products from the reactions. ${ }^{2}$ Data related to granular particle size, abundance of cubic structures, and sizes of cubic crystals are approximate estimates based on randomly imaged sites. The numbers are mainly intended for discussion, and to demonstrate a trend, and are not absolute values. ${ }^{3}$ Obtained from $5 \mu \mathrm{m} \times 5 \mu \mathrm{m}$ SEM images.

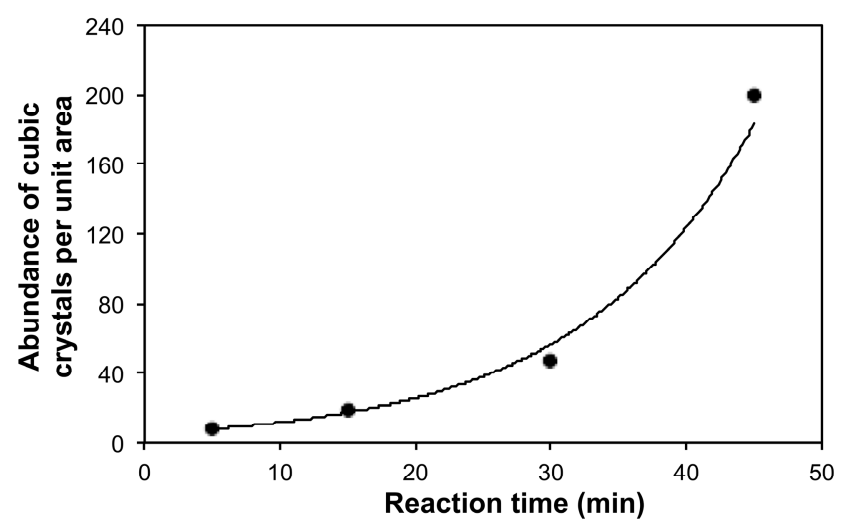

Figure 3. Plot of the abundance of cubic crystal morphology per unit area $\left(25 \mu \mathrm{m}^{2}\right)$ as determined by SEM) in the Cu-SWCNT composites prepared by electroless deposition as a function of reaction time $\left(R^{2}=0.992\right)$.

\subsection{EDX Analysis}

The EDX characterization of the Cu-SWCNT composite samples is summarized in Table 3. As can be seen, there is significant Fe present due to catalyst residue [30-32]. As would be expected, the copper composition is inversely proportional to carbon (and associated $\mathrm{Fe}$ ) content (Figure 4), associated with the deposition of $\mathrm{Cu}$. The EDX analysis of carbon ( $\mathrm{wt} \%$ ) is somewhat close to quantitative with respect to the $w t \%$ of SWCNTs added at high reaction times (Figure 5), after considering the high error bars involved and the estimate made, suggesting that all the SWCNTs in the reaction solution are incorporated into the composite as the reaction proceeds; i.e., samples with a low SWCNT content. This is possible considering that all dispersed solids in the plating bath were collected onto a filter membrane and dried. The high error bars were likely due to the heterogeneity (due to the randomness of the chosen electroless deposition process) of the selected samples and the relatively small proportion of the sample volume analyzed using EDX.

It should be noted that there appears to be an anomaly between the $C$ content for the 30 and 45 min samples (Table 3); however, their values are within the variation between various analysis locations within an individual sample (given as a standard error in Table 3). The observed variations could be due to the local heterogeneity of the samples. Figure 4 shows a plot of elemental composition as a function of the SWCNT concentration within the samples. 
Table 3. Elemental composition (atm.\%) of Cu-SWCNT composites prepared by electroless deposition as determined by EDX ${ }^{1}$.

\begin{tabular}{|c|c|c|c|c|c|c|c|c|c|}
\hline \multirow{2}{*}{$\begin{array}{l}\text { Reaction } \\
\text { Time (min) }\end{array}$} & \multirow{2}{*}{$\begin{array}{l}\text { SWCNT } \\
(w t \%)^{2}\end{array}$} & \multicolumn{2}{|c|}{$\mathrm{C}$} & \multicolumn{2}{|c|}{$\mathbf{O}$} & \multicolumn{2}{|c|}{$\mathrm{Fe}$} & \multicolumn{2}{|c|}{$\mathrm{Cu}$} \\
\hline & & Atm. $\%$ & Wt. $\%$ & Atm. $\%$ & Wt. $\%$ & Atm. $\%$ & Wt. $\%$ & Atm. $\%$ & Wt. $\%$ \\
\hline 5 & 10.6 & $5.03 \pm 1.53$ & $\begin{array}{c}19.79 \pm \\
5.38\end{array}$ & $2.30 \pm 0.22$ & $7.09 \pm 0.86$ & $5.37 \pm 0.50$ & $4.67 \pm 0.26$ & $86.41 \pm 2.47$ & $66.88 \pm 5.90$ \\
\hline 15 & 8.3 & $1.76 \pm 1.70$ & $7.03 \pm 6.71$ & $2.23 \pm 0.77$ & $7.46 \pm 2.14$ & $3.12 \pm 0.66$ & $3.03 \pm 0.38$ & $92.88 \pm 3.04$ & $82.48 \pm 8.78$ \\
\hline 30 & 7.2 & $0.99 \pm 0.63$ & $4.67 \pm 2.83$ & $0.99 \pm 0.35$ & $3.57 \pm 1.11$ & $2.94 \pm 0.11$ & $3.12 \pm 0.20$ & $95.08 \pm 0.90$ & $88.63 \pm 3.76$ \\
\hline 45 & 6.5 & $1.38 \pm 0.49$ & $6.50 \pm 2.16$ & $1.60 \pm 0.10$ & $5.7 \pm 0.29$ & $2.62 \pm 0.17$ & $2.69 \pm 0.20$ & $94.40 \pm 0.55$ & $85.12 \pm 2.19$ \\
\hline
\end{tabular}

${ }^{1}$ Standard error of the mean, $n=3 .{ }^{2}$ The $w t . \%$ values in this column are calculated ideal values based on the mass of SWCNT added to the electroless reaction mixtures, as a function of the final mass of the air-dried products from the reactions.

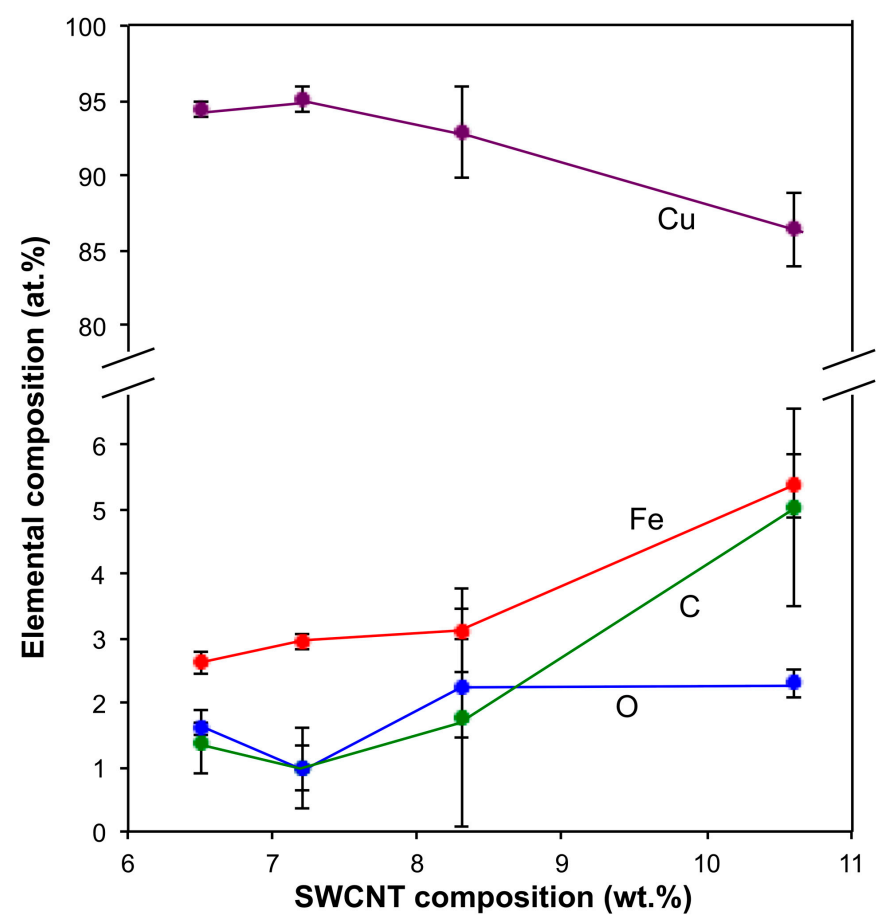

Figure 4. Elemental composition as a function of added SWCNT content (error bars in standard error of mean, $n=3)$.

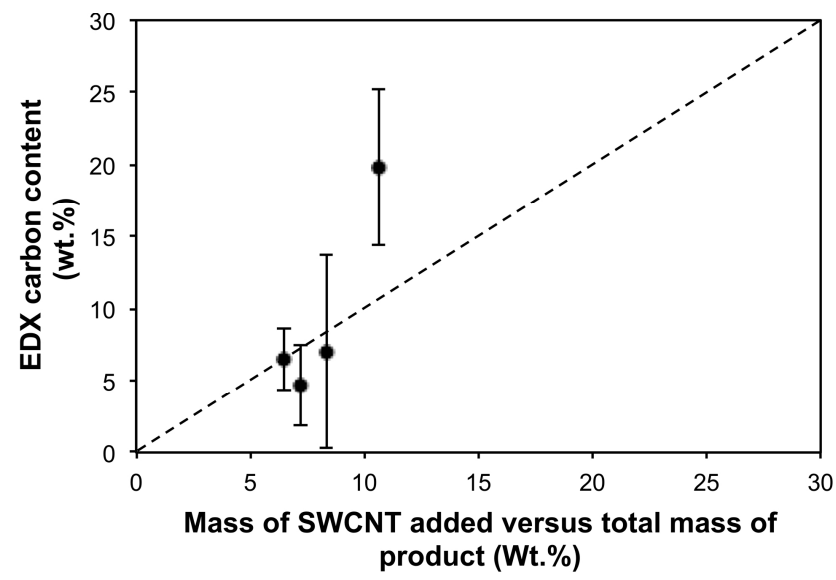

Figure 5. Carbon content (wt.\%) as determined by EDX as a function of the calculated ideal values of SWCNTs (wt.\%) based on the mass of SWCNT added to the electroless reaction mixtures, as a function of the final mass of the air-dried products from the reactions. The dashed line represents an ideal relationship assuming both a homogeneous material and that all the SWCNTs are incorporated into the composite. 
The presence of oxygen could be due to native oxide layers on the copper formed by self-passivation, along with oxygen-containing defect groups on nanotube surfaces [37]. It is also likely that copper oxides may have formed. Prior researchers have reported on the electroless deposition of copper oxides on CNTs [38]. It is interesting to note, however, that the oxygen content does not appear to alter dramatically with SWCNT content (Table 3 and Figure 4). This is in agreement with our observation for the electrodeposition of $\mathrm{Cu}$ on SWCNTs, in which there was initially a drastic drop in oxygen content upon the addition of the CNTs, but no variation in oxygen content was subsequently observed between the composites containing varying concentrations of CNTs [23]. This phenomenon is likely due to a reduction in the oxidation of copper due to the presence of the SWCNTS, coupled with enhanced corrosion potential, reduction of copper, and/or passivation of copper, as was summarized from trends in prior literature [23].

\subsection{XPS Analysis}

Table 4 below gives a summary of the XPS characterization of Cu-SWCNT composites prepared by electroless deposition. Table 5 shows the relative amounts of $\mathrm{Cu}\left(\mathrm{and} / \mathrm{or}_{\mathrm{Cu}} \mathrm{Cu}\right)$ and $\mathrm{Cu}(\mathrm{OH})_{2}$ as determined by fitting the Cu 2p XPS spectra [23]. Please note that SWCNT Wt.\% values in Tables 4 and 5 are calculated values from Table 1 and are used for reference in terms of the actual proportions of SWCNTs physically incorporated into the bulk composite materials, while all other wt.\% and at.\% values in this table are derived from XPS measurements. No clear trends were seen between the various samples in the case of the elemental content or the ratios between elements and the different copper chemical species; i.e., metallic copper (and/or cuprous oxide) and copper(II) hydroxide. However, the main value of XPS was in the detection of the presence of chemical compounds such as copper(II) hydroxide and cuprous oxide. The presence of $\mathrm{Cu}(\mathrm{OH})_{2}$ appears to be a surface (or amorphous) species, given that none is observed by XRD (see Section 3.4), and may have been caused by a reaction between surface copper oxide and atmospheric moisture. The atomic percentages of carbon, as observed in the XPS data, were much higher than in the case of EDX measurements, which is likely due to the local heterogeneity in the samples coupled with the fact that EDX collects data that is more three-dimensionally representative than XPS owing to its greater penetration of a given sample, and since XPS is more of a surface characterization technique.

Table 4. Elemental composition of Cu-SWCNT composites prepared by electroless deposition as determined by XPS.

\begin{tabular}{ccccc}
\hline $\begin{array}{c}\text { Reaction Time } \\
\text { (min) }\end{array}$ & $\begin{array}{c}\text { SWCNT } \\
(\mathbf{w t} \%)\end{array}$ & C (at\%) & O (at\%) & Cu (at\%) \\
\hline 5 & 10.6 & 68.20 & 22.78 & 9.01 \\
15 & 8.3 & 62.82 & 25.37 & 11.79 \\
30 & 7.2 & 71.64 & 21.80 & 6.54 \\
45 & 6.5 & 60.59 & 27.97 & 11.42 \\
\hline
\end{tabular}

${ }^{1}$ The wt.\% values in this column are calculated ideal values based on mass of SWCNT added to the electroless reaction mixtures, as a function of the final mass of the air-dried products from the reactions.

Table 5. Chemical composition of copper species estimated from the relative areas of components within the Cu 2p XPS Spectra of Cu-SWCNT composites prepared by electroless deposition ${ }^{1}$.

\begin{tabular}{cccc}
\hline Reaction Time (min) & ${\text { SWCNT }(\mathbf{w t} \%)^{\mathbf{2}}}^{\mathbf{C u}}$ & $\mathbf{C u}$ and $\mathbf{C u}_{\mathbf{2}} \mathbf{O}(\mathbf{a t} \%)$ & $\mathbf{C u}(\mathbf{O H})_{\mathbf{2}}(\mathbf{a t} \%)$ \\
\hline 5 & 10.6 & 64.26 & 35.73 \\
15 & 8.3 & 64.32 & 35.67 \\
30 & 7.2 & 58.16 & 41.83 \\
45 & 6.5 & 61.67 & 38.32
\end{tabular}

${ }^{1}$ Contributions from $\mathrm{Cu}$ and $\mathrm{Cu}_{2} \mathrm{O}$ are not easily distinguished from each other due to the close proximity of the associated components. ${ }^{2}$ The wt.\% values in this column are calculated ideal values based on the mass of SWCNT added to the electroless reaction mixtures, as a function of the final mass of the air-dried products from the reactions. 


\subsection{XRD Analysis}

The X-ray diffraction (XRD) of the Cu-SWCNT composite samples (Figure 6) reveals crystalline $\mathrm{Cu}$ to be the major component in all cases (Table 6). As is suggested by XPS (see above), there is a minor component of oxide in each sample. The oxide in all the samples containing SWCNTs is $\mathrm{Cu}_{2} \mathrm{O}$ (ICDD \#04-002-3214), and this trend corroborates the observations via XPS analysis presented in the previous section of this paper. Interestingly, the relative percentage of oxide initially decreases with reaction time to $30 \mathrm{~min}$ and then appears to remain essentially constant (Figure 7). It is possible that this change is due to the change in morphology (Figure 1) and increase in cubic crystals versus granular features (Figure 3). As per the XRD data, the crystal grain size of the $\mathrm{Cu}$ generally increases with a longer reaction time, while the $\mathrm{Cu}_{2} \mathrm{O}$ grain size remains small and relatively constant. The SEM image data discussed earlier did not specifically demonstrate an increase in $\mathrm{Cu}$ crystal grain size with reaction time, possibly due to the small sample areas examined under the SEM, while XRD is more of a bulk characterization technique. Hence, grain/crystal sizes and numbers need to be put into context given the random nature of the chosen electroless process and may not be considered as absolute values. While it is obvious that the cubic crystals observed in the SEM are metallic $\mathrm{Cu}$, it is consistent with $\mathrm{XRD}$ and SEM that the granular features are predominantly $\mathrm{Cu}_{2} \mathrm{O}$. This would suggest that the initial deposition of copper onto the raw SWCNTs results in the formation of $\mathrm{Cu}_{2} \mathrm{O}$ in preference to $\mathrm{Cu}$. We have previously observed that the attempted deposition of $\mathrm{Cu}$ nanoparticles (NPs) onto CNTs resulted in tenorite-decorated CNTs (CuO-CNTs) instead [38].

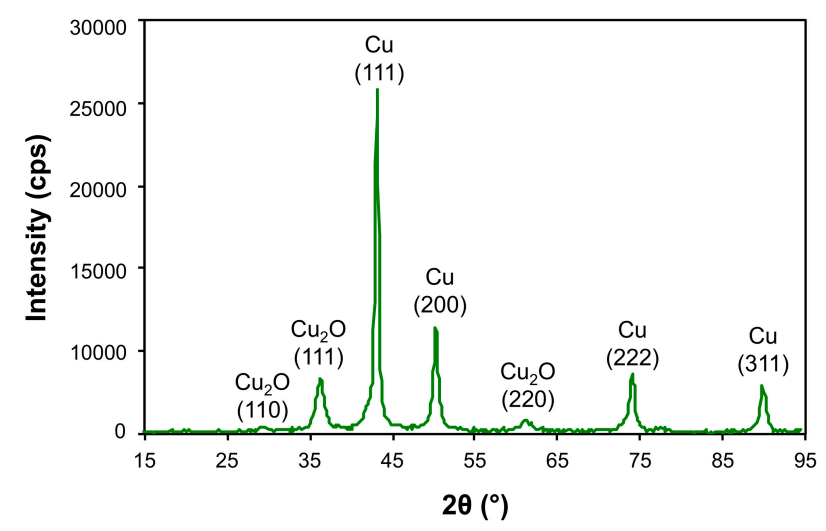

Figure 6. Example of the XRD of a Cu-SWCNT composite prepared by electroless deposition after a reaction of $15 \mathrm{~min}$, showing the presence of $\mathrm{Cu}$ (ICDD \#04-009-2090) and $\mathrm{Cu}_{2} \mathrm{O}$ (ICDD \#04-002-3214).

Table 6. Chemical composition and crystal grain size as determined by XRD for Cu-SWCNT composites prepared by electroless deposition ${ }^{1}$.

\begin{tabular}{cccccc}
\hline \multirow{2}{*}{$\begin{array}{c}\text { Reaction Time } \\
(\mathbf{m i n})\end{array}$} & $\begin{array}{c}\text { SWCNT } \\
(\mathbf{w t} \%)^{\mathbf{2}}\end{array}$ & \multicolumn{2}{c}{ Composition } & \multicolumn{2}{c}{ Crystal Grain Size } \\
\cline { 3 - 6 } & 10.6 & $56.5 \pm 0.3$ & $43.5 \pm 0.3$ & $185 \pm 7$ & $102.6 \pm 0.5$ \\
5 & 8.3 & $59.7 \pm 0.3$ & $40.3 \pm 0.3$ & $192 \pm 5$ & $103.5 \pm 1.3$ \\
15 & 7.2 & $71.6 \pm 0.4$ & $28.4 \pm 0.4$ & $339 \pm 21$ & $90 \pm 2$ \\
30 & 6.5 & $69.2 \pm 0.3$ & $30.8 \pm 0.3$ & $247 \pm 11$ & $119.9 \pm 1.0$ \\
45 & & &
\end{tabular}

${ }^{1}$ Error values given in parentheses. ${ }^{2}$ The wt.\% values in this column are calculated ideal values based on the mass of SWCNT added to the electroless reaction mixtures, as a function of the final mass of the air-dried products from the reactions. 


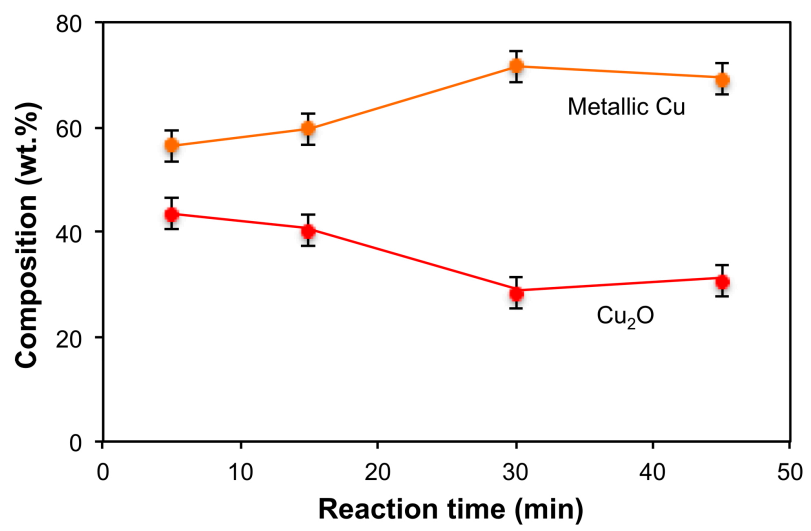

Figure 7. Plot of composition of Cu-SWCNT composites prepared by electroless deposition as determined by XRD.

\subsection{Raman Analysis}

The main purpose of the Raman analysis of the Cu-SWCNT composites prepared in this study is to prove the presence of SWCNTs in the composites. The Raman spectra of the Cu-SWCNTs composites show D and G bands and the radial breathing modes associated with SWCNTs (e.g., Figure 8). The presence of these SWCNT-related signature peaks in the Raman data related to the synthesized composites reliably demonstrate the incorporation of the SWCNTs into the composites. The D band represents the presence of $\mathrm{sp}^{3}$ carbon centers, and the $\mathrm{G}$ band is associated with the $\mathrm{sp}^{2}$ carbon of a pristine SWCNT. The $I_{G}: I_{D}$ ratio is often used as a good indicator of SWCNT quality in bulk samples $[39,40]$. As seen in Table 7 , the $\mathrm{I}_{G}: \mathrm{I}_{\mathrm{D}}$ ratios appeared to decrease slightly as a function of reaction time, although it is clear the values are within the large standard deviation typical of raw SWCNTs [31].

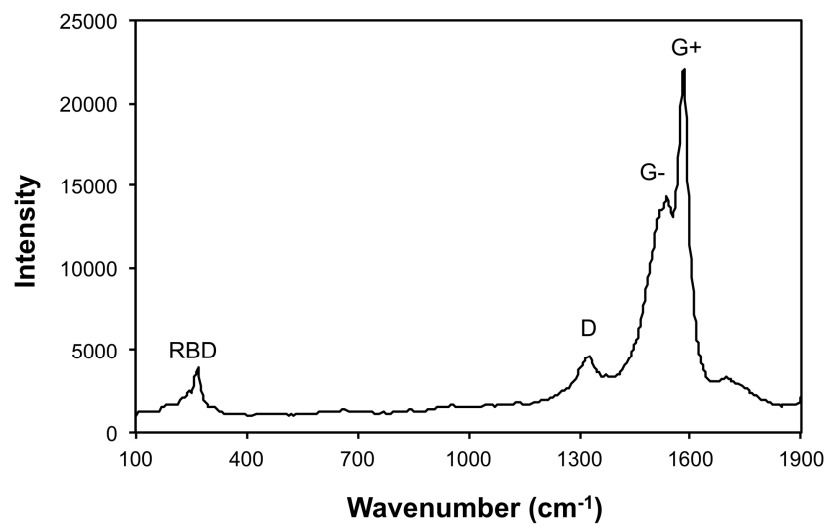

Figure 8. Raman spectra ( $514 \mathrm{~nm}$ ) of the Cu-SWCNT composites prepared by electroless deposition after a reaction time of $30 \mathrm{~min}$.

Table 7. The $\mathrm{I}_{\mathrm{G}}: \mathrm{I}_{\mathrm{D}}$ ratio determined from the Raman spectrum $(514 \mathrm{~nm})$ of $\mathrm{Cu}-\mathrm{SWCNT}$ composites via electroless deposition at a bath temperature of $\sim 90^{\circ} \mathrm{C}^{1}$.

\begin{tabular}{ccc}
\hline Reaction Time (min) & SWCNT $\left(\mathbf{w t} \mathbf{\%}^{\mathbf{2}} \mathbf{~}^{\mathbf{2}}\right.$ & $\mathbf{I}_{\mathbf{G}}: \mathbf{I}_{\mathbf{D}}$ \\
\hline 5 & 10.6 & $4.69(1.65)$ \\
15 & 8.3 & $4.14(1.16)$ \\
30 & 7.2 & $4.13(1.49)$ \\
45 & 6.5 & $3.44(0.95)$ \\
\hline
\end{tabular}

${ }^{1}$ All samples averaged from three regions of the sample, and standard deviations are given in parenthesis. 2 The wt.\% values in this column are calculated ideal values based on the mass of SWCNT added to the electroless reaction mixtures, as a function of the final mass of the air-dried products from the reactions. 


\section{Conclusions}

In summary, an attempt was made to fill in a gap in the literature; to this end, SWCNTs were incorporated into composites with copper, rather than the MWCNTS previously reported in literature. A range of $\mathrm{Cu}$-raw HiPCO SWCNT composite samples were prepared via the electroless method (using polyethylene glycol as a dispersing agent) adapted from procedures published in studies that were more focused on synthesizing nickel-graphite composites. The samples were subjected to characterization using a range of techniques in terms of their morphology, composition, crystallinity, detection of SWCNTs, and ratios between metal impurities such as oxides and hydroxides, in addition to the G:D ratios of the added SWCNTs. The control of the proportion of added raw HiPCO SWCNTs in the electroless composites was achieved by controlling the reaction times. Initially, at low reaction times, the morphology of the obtained electroless $\mathrm{Cu}$-SWCNT composite material was fibrous (mimicking the morphology of the SWCNTs), but this aspect decreased with increased reaction time due to the progressive deposition of excess $\mathrm{Cu}$ that masked the fibrous structure of the SWCNT bundles. Raman data confirmed the presence of SWCNTs in samples based on all sample times, regardless of whether or not the fibrous structures were visible under the SEM, while demonstrating a possible slight (taking into consideration the error bars) reduction in the G:D values with an increase in copper content; i.e., with increased reaction time (even though the error bars were quite high, suggesting a need for SWCNTs of better purity and consistency for future studies). In general, there was a likely inverse proportionality between SWCNT-based (and related iron catalyst material) content in the composites (via the added SWCNTs) and the copper deposited during the electroless process, at a rate that was initially fast and then slowed down. However, oxygen levels were more or less constant regardless of SWCNT content, similar to the case of electroplated Cu-SWCNT composites described in a previous report from our group. XRD analysis confirmed the presence of bulk oxides in the presence of small crystallites (mainly cuprous oxide), while XPS detected the presence of copper(II) hydroxide. An awareness of possible impurities present in as-received raw materials (especially the SWCNTs) and those generated/involved during the synthesis of Cu-SWCNT composites as described in this study, combined with a systematic choice of detailed characterization steps and data analysis, will help to optimize subsequent synthesis and purification efforts, meaning that high-quality metal-nanomaterial composites can be generated for critical electrical applications that require both high electrical conductivity and high ampacity.

Author Contributions: A.R.B. conceptualized the study, wrote, reviewed, and edited the manuscript, and contributed in terms of funding acquisition, project management, supervision, and overall guidance in the study. C.E.G. and K.W. developed the methodology. P.M.V.R. performed the electroless deposition studies, Raman analysis, and contributed to reviewing the paper. C.E.G. and D.R.J. contributed to the XPS characterization and discussion of data. SEM and EDX and related analyses were performed by G.L.E. and B.E.B. B.E.B. also reviewed/edited the paper. XRD analysis was conducted by J.L.

Funding: Funding for this work was provided by the Office of Naval Research (N00014-15-2717), a Sêr Cymru II Welsh Fellowship (C.E.G.), part funded by the European Regional Development Fund (ERDF), and Flexible Integrated Energy Systems (FLEXIS) operations funded by the Welsh European Funding Office (WEFO) through the Welsh Government (A.R.B.).

Acknowledgments: The authors would like to acknowledge the assistance provided by Swansea University AIM Facility, which was funded in part by the EPSRC (EP/M028267/1), and the European Regional Development Fund through the Welsh Government (80708). Thanks are extended to John Marsh of Rice University for administration support and help with project management.

Conflicts of Interest: The authors declare no conflict of interest.

\section{References}

1. Hjortstam, O.; Isberg, P.; Soderholm, S.; Dai, H. Can we achieve ultra-low resistivity in carbon nanotube-based metal composites? Appl. Phys. A 2004, 78, 1175-1179. [CrossRef]

2. Li, Y.; Fahrenthold, E. Quantum conductance of copper-carbon nanotube composites. J. Eng. Mater. Technol. 2018, 140, 031007. [CrossRef] 
3. Han, B.; Guo, E.; Xue, X.; Zhao, Z.; Li, T.; Xu, Y.; Luo, L.; Hou, H. Fabricating and strengthening the carbon nanotube/copper composite fibers with high strength and high electrical conductivity. Appl. Surf. Sci. 2018, 441, 984-992. [CrossRef]

4. Wang, X.; Behabtu, N.; Young, C.C.; Tsentalovich, D.E.; Pasquali, M.; Kono, J. High-ampacity power cables of tightly-packed and aligned carbon nanotubes. Adv. Funct. Mater. 2014, 24, 3241-3249. [CrossRef]

5. Wei, B.Q.; Vajtai, R.; Ajayan, P.M. Reliability and current carrying capacity of carbon nanotubes. Appl. Phys. Lett. 2001, 79, 1172-1174. [CrossRef]

6. Subramaniam, C.; Yamada, T.; Kobashi, K.; Sekiguchi, A.; Futaba, D.N.; Yumura, M.; Hata, K. One hundred fold increase in current carrying capacity in a carbon nanotube-copper composite. Nat. Commun. 2013, 4, 2202. [CrossRef] [PubMed]

7. Subramaniam, C.; Sekiguchi, A.; Yamada, T.; Futaba, D.N.; Hata, K. Nano-scale, planar and multi-tiered current pathways from a carbon nanotube-copper composite with high conductivity, ampacity and stability. Nanoscale 2016, 8, 3888-3894. [CrossRef]

8. Chinnappan, A.; Baskar, C.; Kim, H.; Ramakrishna, S. Carbon nanotube hybrid nanostructures: Future generation conducting materials. J. Mater. Chem. A 2016, 4, 9347-9361. [CrossRef]

9. Chai, G.; Sun, Y.; Sun, J.J.; Chen, Q. Mechanical properties of carbon nanotube-copper nanocomposites. J. Micromech. Microeng. 2008, 18, 035013. [CrossRef]

10. Daoush, W.M.; Lim, B.K.; Mo, C.B.; Nam, D.H.; Hong, S.H. Electrical and mechanical properties of carbon nanotube reinforced copper nanocomposites fabricated by electroless deposition process. Mater. Sci. Eng. A 2009, 513-514, 247-253. [CrossRef]

11. Uddin, S.M.; Mahmud, T.; Wolf, C.; Glanz, C.; Kolaric, I.; Volkmer, C.; Höller, H.; Wienecke, U.; Roth, S.; Fecht, H.-J. Effect of size and shape of metal particles to improve hardness and electrical properties of carbon nanotube reinforced copper and copper alloy composites. Compos. Sci. Technol. 2010, 70, 2253-2257. [CrossRef]

12. Wright, K.D.; Gowenlock, C.E.; Bear, J.C.; Barron, A.R. Understanding the effect of functional groups on the seeded growth of copper on carbon nanotubes for optimizing electrical transmission. ACS Appl. Mater. Interfaces 2017, 9, 27202-27212. [CrossRef] [PubMed]

13. Firkowska, I.; Boden, A.; Vogt, A.-M.; Reich, S. Effect of carbon nanotube surface modification on thermal properties of copper-CNT composites. J. Mater. Chem. 2011, 21, 17541-17546. [CrossRef]

14. Milowska, K.Z.; Burda, M.; Wolanicka, L.; Bristowe, P.D.; Koziol, K.K.K. Carbon nanotube functionalization as a route to enhancing the electrical and mechanical properties of $\mathrm{Cu}-\mathrm{CNT}$ composites. Nanoscale 2019, 11, 145-157. [CrossRef] [PubMed]

15. Hannula, P.-M.; Aromaa, J.; Wilson, B.P.; Janas, D.; Koziol, K.; Forsén, O.; Lundström, M. Observations of copper deposition on functionalized carbon nanotube films. Electrochim. Acta 2017, 232, 495-504. [CrossRef]

16. Xu, C.; Wu, G.; Liu, Z.; Wu, D.; Meek, T.T.; Han, Q. Preparation of copper nanoparticles on carbon nanotubes by electroless plating method. Mater. Res. Bull. 2004, 39, 1499-1505. [CrossRef]

17. Dingsheng, Y.; Yingliang, L. Electroless deposition of $\mathrm{Cu}$ on multiwalled carbon nanotubes. Rare Metals 2006, 25, 237-240.

18. Song, J.L.; Chen, W.G.; Dong, L.L.; Wang, J.J.; Deng, N. An electroless plating and planetary ball milling process for mechanical properties enhancement of bulk CNTs/Cu composites. J. Alloys Compd. 2017, 720, 54-62. [CrossRef]

19. Hwang, H.-J.; Joo, S.-J.; Kim, H.-S. Copper nanoparticle/multiwalled carbon nanotube composite films with high electrical conductivity and fatigue resistance fabricated via flash light sintering. ACS Appl. Mater. Interfaces 2015, 7, 25413-25423. [CrossRef]

20. Arai, S.; Osaki, T. Fabrication of copper/multiwalled carbon nanotube composites containing different sized nanotubes by electroless deposition. J. Electrochem. Soc. 2015, 162, D68-D73. [CrossRef]

21. Arnaud, C.; Lecouturier, F.; Mesguich, D.; Ferreira, N.; Chevallier, G.; Estournès, C.; Weibel, A.; Laurent, C. High strength-high conductivity double-walled carbon nanotube-copper composite wires. Carbon 2016, 96, 212-215. [CrossRef]

22. Raja, P.M.V.; Esquenazi, G.L.; Wright, K.D.; Gowenlock, C.E.; Brinson, B.E.; Alexander, S.; Jones, D.R.; Gangoli, V.S.; Barron, A.R. Aqueous electromigration of single-walled carbon nanotubes and co-electromigration with copper ions. Nanoscale 2018, 10, 19628-19637. [CrossRef] [PubMed] 
23. Raja, P.M.V.; Esquenazi, G.L.; Gowenlock, C.E.; Jones, D.R.; Li, J.; Brinson, B.E.; Barron, A.R. Electrodeposition of Cu-SWCNT composites. C 2019, 5, 38. [CrossRef]

24. Gangoli, V.S.; Raja, P.M.V.; Esquenazi, G.L.; Barron, A.R. The safe handling of bulk low-density nanomaterials. SN Appl. Sci. 2019, 1, 644.

25. Gowenlock, C.E.; Gomez, V.; McGettrick, J.D.; Andreoli, E.; Barron, A.R. Surface-initiated growth of copper using isonicotinic acid-functionalized aluminum oxide surfaces. J. Coat. Technol. Res. 2017, 14, 195-205. [CrossRef]

26. Caturla, F.; Molina, F.; Molina-Sabio, M.; Rodríguez-Reinoso, F.; Esteban, A. Electroless plating of graphite with copper and nickel. J. Electrochem. Soc. 1995, 142, 4084-4090. [CrossRef]

27. Baker, E.M. Method of nickel plating. U.S. Patent 2,312,517, 2 March 1943.

28. Brenner, A.; Riddell, G.E. Nickel plating by chemical reduction. U.S. Patent 2,312,517, 5 December 1950.

29. Zaitoun, M.A.; Lin, C.T. Chelating behavior between metal ions and EDTA in sol-gel matrix. J. Phys. Chem. $B$ 1997, 101, 1857-1860. [CrossRef]

30. Lee, S.M.; Raja, P.M.V.; Esquenazi, G.L.; Barron, A.R. Effect of raw and purified carbon nanotubes and iron oxide nanoparticles on the growth of wheatgrass prepared from the cotyledons of common wheat (triticum aestivum). Environ. Sci. Nano 2018, 5, 103-114. [CrossRef]

31. Zhang, K.S.; Pham, D.; Lawal, O.; Ghosh, S.; Gangoli, V.S.; Smalley, P.; Kennedy, K.; Brinson, B.; Billups, W.E.; Hauge, R.; et al. Overcoming catalyst residue inhibition of the functionalization of single-walled carbon nanotubes via the Billups-Birch reduction. ACS Appl. Mater. Interfaces 2017, 9, 37972-37980. [CrossRef]

32. Gomez, V.; Irusta, S.; Adams, W.W.; Hauge, R.H.; Dunnill, C.W.; Barron, A.R. Enhanced carbon nanotubes purification by physic-chemical treatment with microwave and $\mathrm{Cl}_{2}$. RSC Adv. 2016, 6, 11895-11902. [CrossRef]

33. Gangoli, V.S.; Kazimierska, E.; Barnett, C.J.; Barron, A.R. Method for the improved purification and increased electrical conductivity of carbon nanomaterials. U.S. Patent Application 62,882,752, 16 July 2019.

34. Plana, D.; Campbell, A.I.; Patole, S.N.; Shul, G.; Dryfe, R.A.W. Kinetics of electroless deposition: The copper-dimethylamine borane system. Langmuir 2010, 26, 10334-10340. [CrossRef] [PubMed]

35. Loscutova, R.; Barron, A.R. Coating single-walled carbon nanotubes with cadmium chalcogenides. J. Mater. Chem. 2005, 15, 4346-4353. [CrossRef]

36. Sharma, R.; Agarwala, R.C.; Agarwala, V. Development of copper coatings on ceramic powder by electroless technique. Appl. Surf. Sci. 2006, 252, 8487-8493. [CrossRef]

37. Ogrin, D.; Chattopadhyay, J.; Sadana, A.K.; Billups, E.; Barron, A.R. Epoxidation and deoxygenation of single-walled carbon nanotubes: Quantification of epoxide defects. J. Am. Chem. Soc. 2006, 128, 11322-11323. [CrossRef] [PubMed]

38. Rudd, J.A.; Gowenlock, C.E.; Gomez, V.; Kazimierska, E.; Al-Enizi, A.M.; Andreoli, E.; Barron, A.R. Solvent-free microwave-assisted synthesis of tenorite nanoparticle-decorated multi-walled carbon nanotubes as precursors for ultra-conductive wire. J. Mater. Sci. Technol. 2019, 35, 1121-1127. [CrossRef]

39. Hussain, S.; Pal, A.K. Incorporation of nanocrystalline silver on carbon nanotubes by electrodeposition technique. AIP Conf. Proc. 2008, 1063, 98-104.

40. Dresselhaus, M.S.; Pimenta, M.A.; Ecklund, P.C.; Dresselhaus, G. Raman Scattering in Materials Science; Springer: Berlin, Germany, 2000.

(C) 2019 by the authors. Licensee MDPI, Basel, Switzerland. This article is an open access article distributed under the terms and conditions of the Creative Commons Attribution (CC BY) license (http://creativecommons.org/licenses/by/4.0/). 